\title{
Numerical investigation of natural convection with the presence of surface roughness or complex geometries
}

\author{
Mohamed Elamine GHEDHAB ${ }^{1,2, *}$, Ikram EL ABBASSI ${ }^{2}$, Rafik ABSI $^{2}$ Yannick MELINGE ${ }^{1}$ \\ ${ }^{1}$ Laboratoire de Mécanique et Matériaux du Génie Civil (L2MGC), Université de Cergy-Pontoise, France \\ ${ }^{2}$ Laboratoire de Recherche en Eco-innovation Industrielle et Energétique (LR2E), ECAM-EPMI, France
}

\begin{abstract}
This paper represents a numerical study of mixed convection conduction in enclosed twodimensional square cavity differentially heated. Complex geometries are designed on a vertical partitioned wall, those geometries are applied to building facades aiming to improve the overall energy performance of the building. Our numerical results are based on the resolution of coupled momentum and energy equations using Ansys Fluent. Numerical study has been performed for different values of the Rayleigh number. Triangular geometry element will be considered in the middle wall. Finally, temperature profile and streamlines will be analyzed to examine the influence of this studied shape.
\end{abstract}

\section{INTRODUCTION}

Conjugate mixed convection in enclosed cavities differentially heated represents a longstanding challenge that has been widely studied last decades due to its abundant applications in several engineering and natural processes. This appears in building applications, nuclear reactor or cooling of electronic devices, solar thermal systems, internal combustion engine and chemical reactors [1], [2], [3].

Previous researches on conjugate mixed convection have considered the thickness as potential factor that can impact the steady state behavior of heat transfer. Among those studies, Acharya \& Tsang [4] studied the impact of the partitioned wall presence in cavities and the inclination of the enclosed cavities, they found that those two parameters change the position of the maximum Nusselt number (Nu). Kahveci [5] investigated numerically the presence of a vertical partition with various finite thickness and different positions, on natural convection inside square cavity in laminar case using polynomial based differential quadrature method. Subsequently, he found that when the position of the wall is more near to the middle, the value of $\mathrm{Nu}$ decreases progressively to achieve a constant. In addition, another study has been carried out by the same author[6], where he inspected the effect of the aspect ratio in rectangular enclosed partitioned cavity, and he noted that when the aspect ratio $\mathrm{A}=1$, the Rayleigh number $\mathrm{Ra}=10^{4}$ and $10^{5}$, the heat transfer is better than lower values of A. Furthermore, Oztop et al. [7] examined laminar natural convection in partially heated divided enclosure, in presence of two different fluids, air and water, they founds that the heat transfer is affected by the location of the middle wall. Recently, Mehdi Khatamifar et al. [8] examined the effects of partition position and thickness on the unsteady-state conjugate natural convection heat transfer in a differentially heated partitioned enclosure over a wide range of Rayleigh numbers, he showed that when the Rayleigh number is increasing the Nusselt number decrease.

However, to our best knowledge, very few publications can be available in the literature that address the issue of wall shape, especially in the building applications. In this context, the main purpose of this study is to improve the energy performance and thermal comfort for the users generally and residents particularly. This improvement will be reached by handling the effect of the vertical wall shape on the steady heat exchange in the conjugate mixed convection of the enclosed square cavities.

\section{PROBLEM FORMULATION}

The physical models under study are shown in Fig 1 and Fig 2. They represent two different wall geometries of two-dimensional partitioned square cavity with finite length $\mathrm{H}$ where $(\mathrm{H}=\mathrm{W})$. We studied the case of square enclosure cavity with $\mathrm{A}=1$. The top and bottom walls of the cavity are considered as adiabatic walls. The left and right vertical walls are isothermal fixed at THot and TCold respectively $\left(\mathrm{T}_{\text {Hot }}>\mathrm{T}_{\text {Cold }}\right)$. The wall thickness is fixed at $0.10 \mathrm{~m}$. Given that the fluid considered in this contribution is the air, the Prandtl number is equal to $\mathrm{Pr}$ $=v / \alpha=0.71$.

The gravity act is in negative y direction. The partition wall is heat conducting, with finite thickness.

To refer to the large-scale constructions, the concrete is chosen as a solid material with following physical properties: density $\rho=2000 \mathrm{~kg} / \mathrm{m} 3$, thermal conductivity $\lambda=1.263 \mathrm{~W} / \mathrm{m} . \mathrm{K}$ and specific heat capacity $\mathrm{Cp}=1000$ $\mathrm{J} / \mathrm{kg} . \mathrm{k}$.

\footnotetext{
* Corresponding author: author@e-mail.org
} 


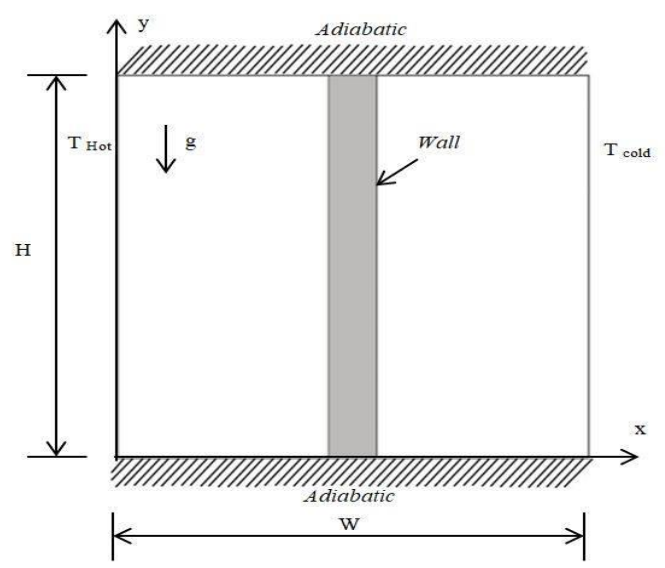

Fig. 1. Schematic representation of the $1^{\text {st }}$ case with vertical smooth wall.

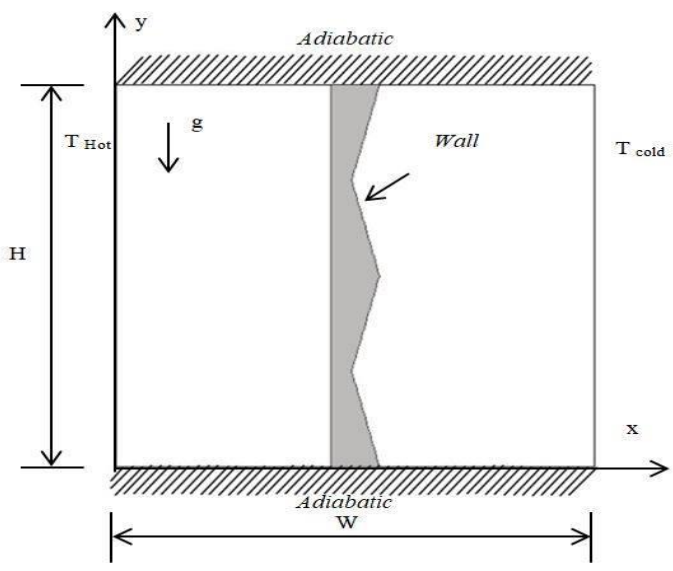

Fig. 2. Schematic representation of the 2 nd case with forms in the wall

Presuming that the fluid is incompressible, Newtonian and the flow is in the steady-state, the fluid flow in both sides of the cavity is represented by the following 2D Naviers-Stokes equations, taking into account the approximation of Boussinesq for buoyancy forces, with the temperature equation in Cartesian coordinates presented below:

$$
\begin{aligned}
& \frac{\partial u}{\partial x}+\frac{\partial v}{\partial y}=0 \\
& u \frac{\partial u}{\partial x}+v \frac{\partial u}{\partial y}=\frac{1}{\rho}\left[-\frac{\partial p}{\partial x}+\mu\left(\frac{\partial^{2} u}{\partial x^{2}}+\frac{\partial^{2} u}{\partial y^{2}}\right)\right] \\
& u \frac{\partial v}{\partial x}+v \frac{\partial v}{\partial y}=\frac{1}{\rho}\left[-\frac{\partial p}{\partial y}+\mu\left(\frac{\partial^{2} v}{\partial x^{2}}+\frac{\partial^{2} v}{\partial y^{2}}\right)\right. \\
& +\rho g \beta \Delta T \\
& u \frac{\partial T}{\partial x}+v \frac{\partial T}{\partial y}=\alpha\left(\frac{\partial^{2} T}{\partial x^{2}}+\frac{\partial^{2} T}{\partial y^{2}}\right)
\end{aligned}
$$

Where $u, v$ represent the velocity components in $\mathrm{x}$ and $\mathrm{y}$ directions; $\mathrm{p}$ and $\mathrm{T}$ denotes the pressure and the temperature; $\rho, \mu, \alpha, g$ and $\beta$ are respectively the density, viscosity, thermal diffusion, gravity and the thermal expansion.
To stand out the governing dimensionless parameters, the following dimensionless variables are applied to the previous four equations:

$$
\begin{gathered}
X=\frac{x}{H} ; \quad Y=\frac{y}{H} ; \quad U=\frac{u}{U_{\text {ref }}} ; \quad V=\frac{v}{U_{\text {ref }}} \\
\theta=\frac{T-T_{C}}{T_{H}-T_{C}} ; \quad P=\frac{p}{\rho U_{\text {ref }}^{2}} ;
\end{gathered}
$$

Considering that $U_{\text {ref }}=\sqrt{g \beta \Delta T L}$ is the characteristic speed and $\theta$ is the dimensionless temperature, the governing flow equations are presented as follow:

$$
\begin{aligned}
& \frac{\partial U}{\partial X}+\frac{\partial V}{\partial Y}=0 \\
& U \frac{\partial U}{\partial X}+V \frac{\partial U}{\partial Y}=-\frac{\partial P}{\partial X}+\operatorname{Pr}\left(\frac{\partial^{2} U}{\partial X^{2}}+\frac{\partial^{2} U}{\partial Y^{2}}\right) \\
& U \frac{\partial V}{\partial X}+V \frac{\partial V}{\partial Y}=-\frac{\partial P}{\partial Y}+\operatorname{Pr}\left(\frac{\partial^{2} U}{\partial X^{2}}+\frac{\partial^{2} V}{\partial Y^{2}}\right) \\
&+\operatorname{RaPr} \cdot \theta \\
& U \frac{\partial \theta}{\partial X}+V \frac{\partial \theta}{\partial Y}=\alpha\left(\frac{\partial^{2} \theta}{\partial X^{2}}+\frac{\partial^{2} \theta}{\partial Y^{2}}\right)
\end{aligned}
$$

The Rayleigh number $R a=\frac{g \beta \Delta T L^{3}}{v \alpha}$ allows to define the flow regime, in our laminar case $\left(R a \leq 10^{6}\right)$, the heat conduction part is governed by:

$$
\frac{\partial^{2} T}{\partial x^{2}}+\frac{\partial^{2} T}{\partial y^{2}}=0
$$

The boundary conditions are considered as:

-The velocity components are fixed to $u=v=0$ on walls surface's;

-Top and bottom walls are set as adiabatic;

-A constant temperatures $\mathrm{T}_{\text {Hot }} / \mathrm{T}_{\text {Cold }}$ are applied in right and left wall assuming that $\left(\mathrm{T}_{\mathrm{Hot}}>\mathrm{T}_{\text {Cold }}\right)$.

-For the middle wall, "coupled thermal condition" is taken in both sides [9].

\section{NUMERICAL APPROACH}

To resolve the previous formulations, the finite volume method integrated in the commercial solver Fluent is used. The velocity-pressure coupling is handled by the SIMPLE algorithm, the discretization schemes are fixed in the second order and the pressure-based solver is done by PRESTO. The obtained results will be presented in the next section.

In order to validate our model, we have considered a square cavity without the middle wall, and compared the results with the benchmark [10] of natural convection (Table1), using the Average Nusselt number values (eq.10) for laminar Rayleigh.

$$
N u_{\text {Ave }}=\int_{0}^{1} N u_{\text {local }} d Y
$$


Table 1.Validation of numerical simulation with benchmark

\begin{tabular}{|c|c|c|c|c|}
\hline & \multicolumn{4}{|c|}{$\mathrm{Ra}$} \\
\cline { 2 - 5 } & $10^{3}$ & $10^{4}$ & $10^{5}$ & $10^{6}$ \\
\hline Mesh size & 0.025 & 0.025 & 0.0125 & 0.0125 \\
\hline $\mathrm{Nu}_{\text {ref }}$ & 1.116 & 2.234 & 4.510 & 8.817 \\
\hline $\mathrm{Nu}_{\text {sim }}$ & 1.126 & 2.283 & 4.594 & 9.074 \\
\hline Error & $0.86 \%$ & $2.20 \%$ & $1.85 \%$ & $2.91 \%$ \\
\hline
\end{tabular}

\section{RESULTS AND DISCUSSIONS}

After validating the natural convection case, several simulations are carried out for different values of Rayleigh number (from $10^{3}$ to $10^{6}$ ), with the presence of partitioned wall in two shapes presented before.

The mesh used for the first configuration has a rectangular form with equal sizes in the $\mathrm{x}$ and $\mathrm{y}$-axis $(\Delta x=\Delta y)$

The difference between the first and the second configurations lies in the meshing technique, when for the second one, the mesh in right side of the cavity was done by hybrid method seeing that we have triangular forms in the right façade.

For different situations with distinct values of $\mathrm{Ra}$, our target is to examine the geometry impact on the variation of the heat transfer and the fluid flow.

From figure 3 and figure 4 , it can be seen that the studied geometry does not impact the air isothermal contours in the left side of partitioned wall for low value of Rayleigh number $\left(10^{3}\right.$ and $\left.10^{4}\right)$, this can be explained by the fact that flow regime is considered as laminar. Moreover, a slight difference in those contours of the temperature near to the wall with modified geometry is notable.
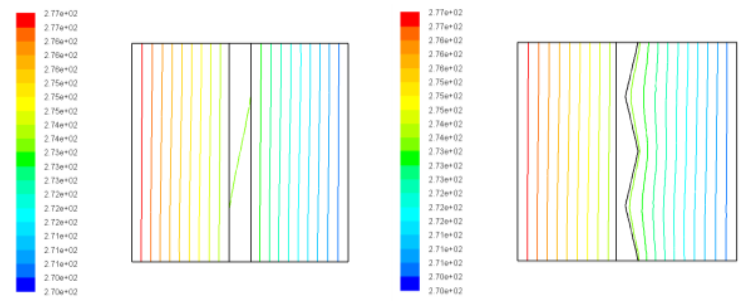

$\mathrm{Ra}=10^{3}$
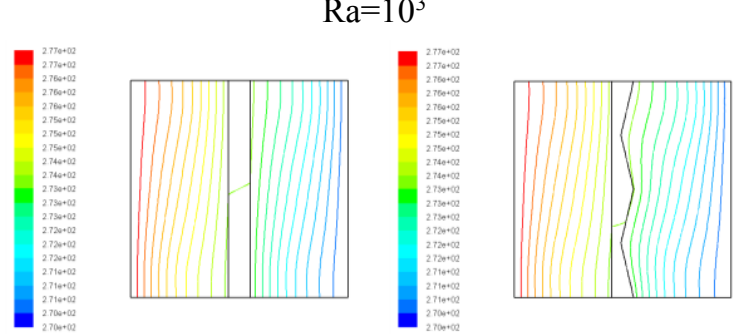

$\mathrm{Ra}=10^{4}$
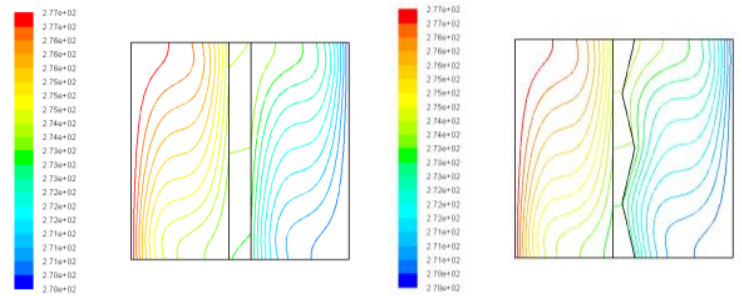

$\mathrm{Ra}=10^{5}$
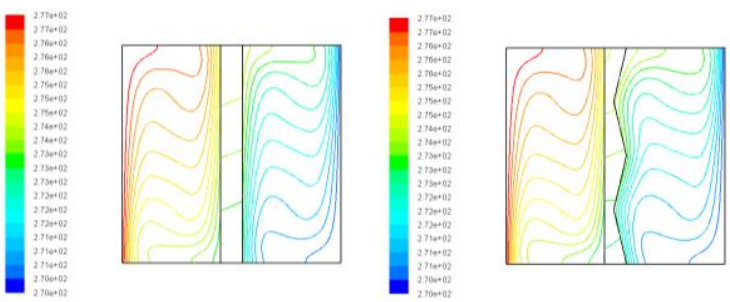

$\mathrm{Ra}=10^{6}$

Fig. 3. Isothermal contours inside the cavities

The temperatures of the profiles shown in Figures 5 and 6 are plotted on the left vertical side of the middle wall, taking into account two different values of $\mathrm{Ra}\left(10^{4}\right.$ and $\left.10^{6}\right)$, since each aims to compare the temperature of the profile for the two cases considered. For Rayleigh $10^{4}$, we found that there is a difference in the top of the wall, and the profile is asymmetrical against the mean temperature, this gap is due to the modified boundary layer for the second case.
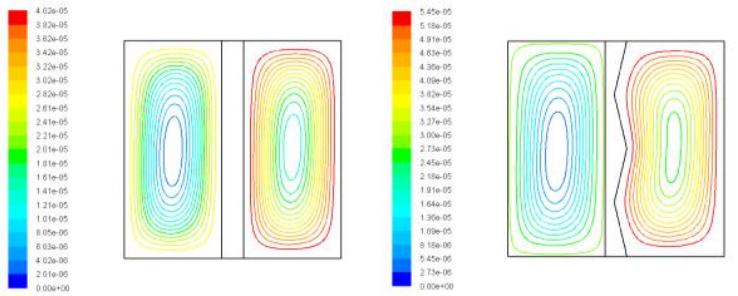

$\mathrm{Ra}=10^{4}$
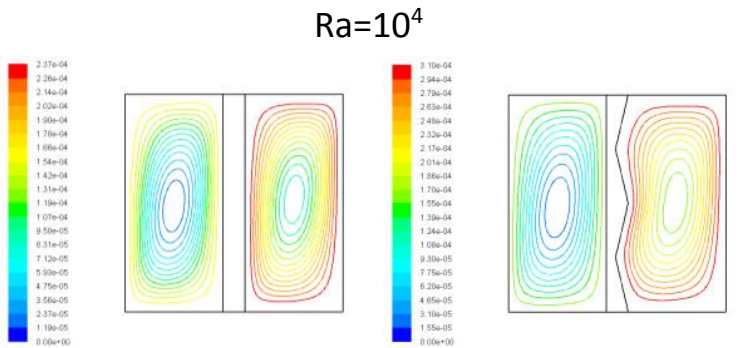

$\mathrm{Ra}=10^{5}$
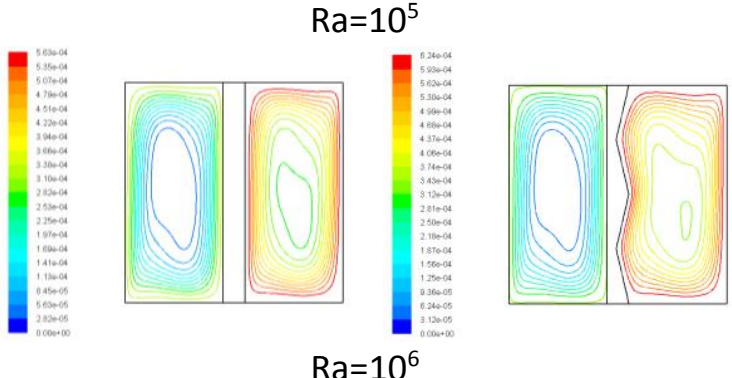

Fig. 4. Streamlines contours

For the figure 6, both profiles has symmetrical form in relation to the average temperature with a difference in the 
top and the bottom of the wall, in this regime of Rayleigh $10^{6}$, the thickness of the boundary layer is less important, and the air flow is more important.

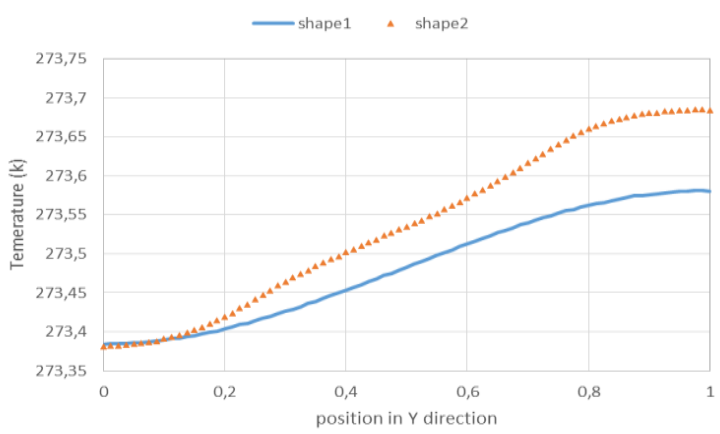

Fig. 5. Temperature profile in left side $\mathrm{Ra}=10^{4}$

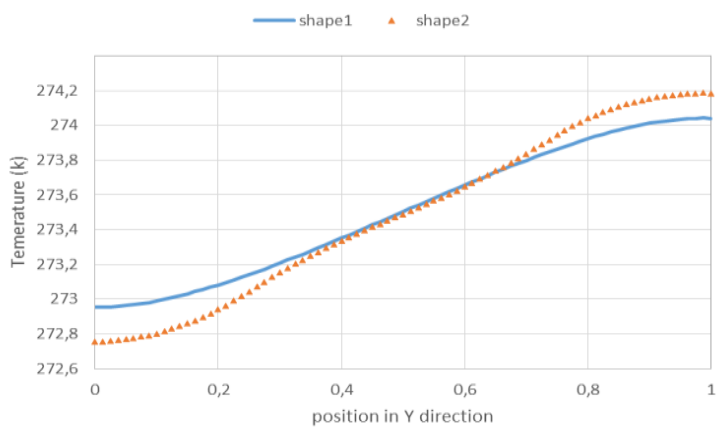

Fig. 6. Temperature profile in left side at $\mathrm{Ra}=10^{6}$

\section{CONCLUSION}

In this paper, a numerical investigation has been carried out in order to evaluate the effect of the partitioned wall shape of an enclosed square cavity differentially heated on the heat transfer. Different values of the Rayleigh number ranging from $10^{3}$ to $10^{6}$ have been considered given that solely the laminar regime of the flow is taking into account. The obtained results showed that the shape of the conductive walls has an impact on the temperature profiles and streamlines especially near to the middle wall. As future works, we intend to test other complex forms in turbulent regime and the presence of surface roughness taking into count the same surface of the middle wall. In addition, we will study the thermal interaction between external wall and internal one.

\section{REFERENCES}

1. Y. Varol, H.F. Oztop, A. Koca, Int. Commun. Heat Mass Transfer, 37, 182 (2010).

2. A. Missoum, M. Elmir, A. Belkacem, M. Nabou, B. Draoui, Energy Procedia, 36, 834 (2013).

3. M. Foruzan Nia, S.A. Gandjalikhan Nassab, A.B. Ansari, Int. J. Therm. Sci., 128, 94 (2018).

4. S. Acharya, C. H. Tsang, Numer. Heat Transfer 8 , 407 (1985).

5. K. Kahveci, Int. J. of Numer. Meth. for Heat \& Fluid Flow, 17, 439 (2007).
6. K. Kahveci, Numer. Heat Transfer, Part A, 51, 979 (2007).

7. H.F. Oztop, Y. Varol, A. Koca, Int. J. Heat Mass Transfer, 52, 5909 (2009).

8. M. Khatamifar, W. Lin, S. Armfield, Davi, Int. Commun. Heat Mass Transfer, 81, 92-103 (2017).

9. ANSYS Fluent 15.0 user's guide.

10. G. De Vahl Davis, Int. J. Numer. Methods Fluids, 3, 249 (1983). 